\section{Spectrum of latent tuberculosis - existing tests cannot resolve the underlying phenotypes: authors' reply}

Clifton E. Barry 3rd, Helena I. Boshoff, Véronique Dartois, Thomas Dick, Sabine Ehrt, JoAnne Flynn, Dirk Schnappinger, Robert J. Wilkinson \& Douglas Young

We agree with the comments in response to our Review (The spectrum of latent tuberculosis: rethinking the biology and intervention strategies. Nature Rev. Microbiol. 7, 845-855 (2009)) $)^{1}$ from Pai (Spectrum of latent tuberculosis - existing tests cannot resolve the underlying phenotypes. Nature Rev. Microbiol. 8, 19 Jan 2010 (doi:10.1038/nrmicro2236-c1)) ${ }^{2}$ stating that current diagnostic tests do not address the challenge of identifying subgroups of individuals in the latent tuberculosis (TB) spectrum who would benefit most from preventive therapy. Indeed, we hope that the framework set out in our Review will help to target efforts towards the development of improved diagnostic tools. The strategy of serial IGRA testing as proposed by Pai is likely to be one element in progressing towards this goal, although broadening the range of cytokines beyond a reliance solely on interferon- $\gamma$ may well be important. A key limitation to the time-dependent approach that is illustrated by Pai is that accurate information about the time of initial exposure is unavailable in most endemic settings. Parallel studies based on experimental challenge in the non-human primate model may be helpful in establishing a clear time course for the correlation of immune markers with microbiological and clinical changes.

Clifton E. Barry 3rd and Helena I. Boshoff are at the Tuberculosis Research Section, Laboratory of Clinical Infectious Diseases, National Institute of Allergy and Infectious Diseases, National Institutes of Health, Bethesda, Maryland 20892, USA.
Véronique Dartois and Thomas Dick are at the Novartis Institute for Tropical Diseases, 10 Biopolis Road, \#05-01 Chromos, Singapore 138670.

Sabine Ehrt and Dirk Schnappinger are at the Department of Microbiology and Immunology, Weill Cornell Medical College, New York, New York 10065, USA.

JoAnne Flynn is at the Department of Molecular Genetics and Biochemistry, Biomedical Science Tower, University of Pittsburgh School of Medicine, Pittsburgh, Pennsylvania 15261, USA.

Robert J. Wilkinson is at the Division of Mycobacterial Research, Medical Research Council National Institute for Medical Research, London NW7 1AA, UK, at the Centre for Molecular Microbiology and Infection and Division of Medicine, Imperial College, London SW7 2AZ, UK and at the Institute of Infectious Diseases and Molecular Medicine, University of Cape Town, Observatory 7925, South Africa.

Douglas Young is at the Division of Mycobacterial Research, Medical Research Council National Institute for Medical Research, London NW7 1AA, UK and at the Centre for Molecular Microbiology and Infection and Division of Medicine, Imperial College, London SW7 2AZ, UK.

Correspondence to D.Y.e-mail: d.young@imperial.ac.uk doi:10.1038/nrmicro2236-c2 Published online 19 January 2010

1. Barry, C. E. 3rd et al. The spectrum of latent tuberculosis: rethinking the biology and intervention strategies. Nature Rev. Microbiol. 7, 845-855 (2009).

2. Madhukar Pai. Spectrum of latent tuberculosis existing tests cannot resolve the underlying phenotypes. Nature Rev. Microbiol. 8, 19 Jan 2010 (doi:10.1038/nrmicro2236-c1).

Competing interests statement

The authors declare no competing financial interests. 